\title{
A double success story: The international cooperation to build the new ICRF antennas on ASDEX Upgrade and the results obtained.
}

\author{
H. Fuenfgelder ${ }^{1}$, D. Aguiam ${ }^{6}$, O. D’Arcangelo ${ }^{3}$, V. Bobkov ${ }^{1}$, S. Ceccuzzi ${ }^{3}$, Y. Chen ${ }^{4}$, H. Faugel ${ }^{1}$, \\ Jacquot $^{1}$, R. Maggiora ${ }^{5}$, A. Mancini ${ }^{3}$ D. Milanesio ${ }^{5}$, J.-M. Noterdaeme ${ }^{1,2}$, R. Ochoukov ${ }^{1}$,

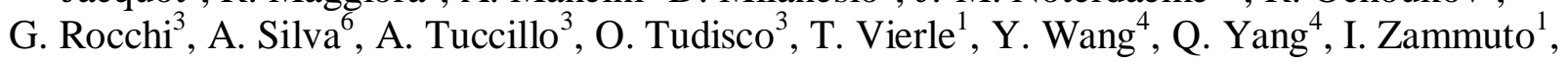 \\ the ASDEX Upgrade ${ }^{1}$ and Eurofusion MST1 Teams ${ }^{1}$ \\ ${ }^{1}$ Max-Planck-Institut für Plasmaphysik, Boltzmannstr. 2, D-85738 Garching, Germany \\ ${ }^{2}$ Ghent University, Applied Physics Department, B-7000 Gent, Belgium \\ ${ }^{3}$ ENEA, Dipartimento FSN, C. R. Frascati, via E. Fermi 45, 00044 Frascati ( Roma), Italy \\ ${ }^{4}$ ASIPP, Institute of Plasma Physics, Chinese Academy of Sciences, Hefei, China \\ ${ }^{5}$ Politecnico de Torino, Torino, Italy \\ ${ }^{6}$ Instituto de Plasmas e Fusão Nuclear, IST, 1049-001 Lisboa, Portugal
}

\begin{abstract}
New ICRF (Ion Cyclotron Range of Frequency) antennas, targeted to reduce impurity generation in the all-metal ASDEX Upgrade were designed and fabricated at different sites in an international cooperation between IPP, ASIPP (Hefei, China) and ENEA (Frascati, Italy). Installation and test were done by a multinational team at IPP. The antenna further integrates a powerful reflectometer system, designed and built by ENEA and IST (Portugal). The project, with the cooperation of four institutes in four countries on two continents was successfully completed within budget and schedule boundaries. Test results, gathered during recent experiment campaign, reveal that the new antennas have achieved their goal: a significant reduction of ICRF heating induced W impurity generation.
\end{abstract}

Keywords: ICRF, ASDEX Upgrade, antenna

\section{Rational for new antenna}

Enhanced impurity production has often accompanied experiments using ICRF (Ion Cyclotron Range of Frequency) as heating method. Positive effects, such as the capability to deposit the power centrally even at high density and thereby reduce the central impurity accumulation, were wiped out in the all-metal ASDEX Upgrade when the antenna limiters were also coated with $\mathrm{W}$ [1]. The hypothesis that this enhanced interaction is due to RF sheaths - possibly a consequence of currents induced at undesirable locations - has, up to now, not been invalidated. A new 3-strap antenna for ASDEX Upgrade [2], targeted to lower these RF sheaths by canceling these undesirable currents induced in the antenna frame, has been designed by IPP based on 3D modeling and intensive simulation, done in cooperation between IPP and ENEA.

\section{Antennas in service up to 2014, Fig. 1}

Four 2-strap antennas, installed in 1992, with various enhancements since, have been in service for ASDEX Upgrade. The straps of the 2-strap antenna were fed with identical power but $180^{\circ}$ phase difference. This results in significant RF image currents at the antenna frame and electric fields at the antenna limiters.
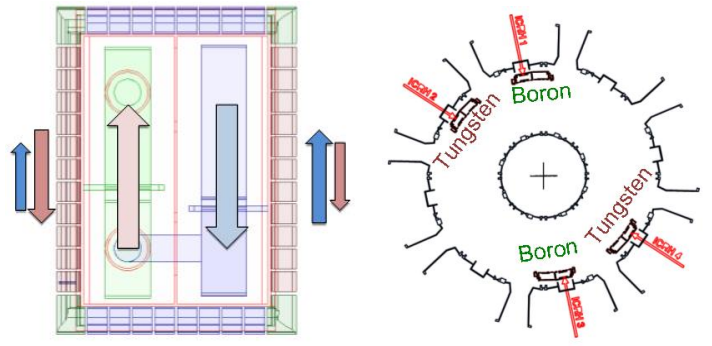

Fig.1: Antenna principle and arrangement in service up to 2014. Since 2011 two antennas have Boron coated limiters and two Tungsten coated ones.

\section{New antenna concept}

The new antenna concept, based on comprehensive simulations, is using three straps $[2,3]$. The central strap and the two outer straps can be fed independently in amplitude and phase. The feeding can thus be optimized to cancel out, as much as possible, currents induced in the antenna frame (Fig. 2).

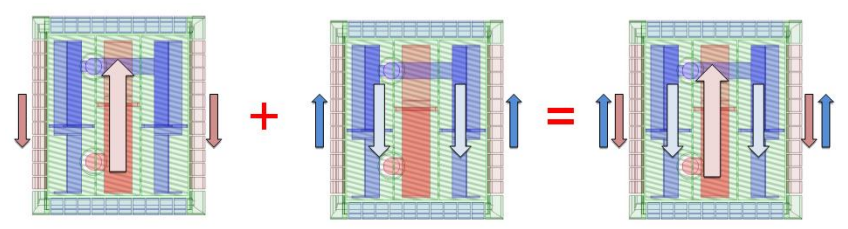

Fig. 2: New antenna concept 


\section{Project work split}

To share experience and cost, and to bundle resources, a strong international cooperation to equip AUG with two new antennas as well as associated diagnostics was formed. The official start of the project, with the signature of a cooperation agreement, was in June 2012, with informal contacts having started a year earlier.

ASIPP took over the responsibility for the 2D manufacturing drawings, based on IPP's 3D drawings, the validation of material and manufacturing technologies as well as the manufacturing of all stainless steel components for two antennas.

ENEA took over responsibility for the 2D manufacturing drawings, based on IPP's 3D drawings, the validation of material and manufacturing technologies as well as the manufacturing of all special material components (e.g. cooling frame and Faraday screen) for two antennas. Fig. 3 shows stainless steel components and complete antenna including the waveguides for the reflectometry.
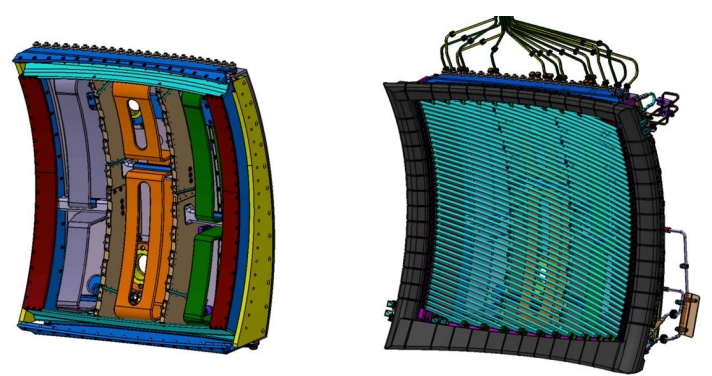

Fig.3: Stainless steel parts (left) and complete antenna (right)

\section{Diagnostics}

To measure density profiles in front of new ICRF antenna, a multi-channel reflectometer was specified: 10 microwave antenna pairs at adequate positions within and around the ICRF antenna, as shown in Fig. 4, with seven antenna pairs routed to the outer world at a time. A three channel FM-CW radar system, covering $40 \mathrm{GHz}$ to $68 \mathrm{GHz}$ with associated data acquisition system permits simultaneous density profile measurements at three selectable positions. Responsibility for above reflectometry system was taken over by ENEA teamed up with IST. [7]

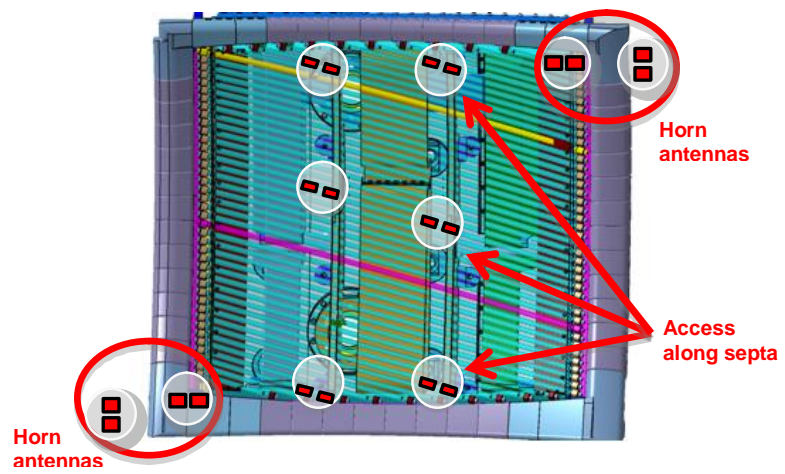

Fig. 4: Positions of reflectometry antennas
To support the characterization of ICRF wave generation [6] and the interaction with magnetized plasma a new Bdot probe-based diagnostic has been installed by IPP, as shown in Fig. 5. The diagnostic consists of a fieldaligned array of High frequency B-dot (HFB) probes with supporting electronics and data acquisition. In addition a neutral pressure measurement device was installed.

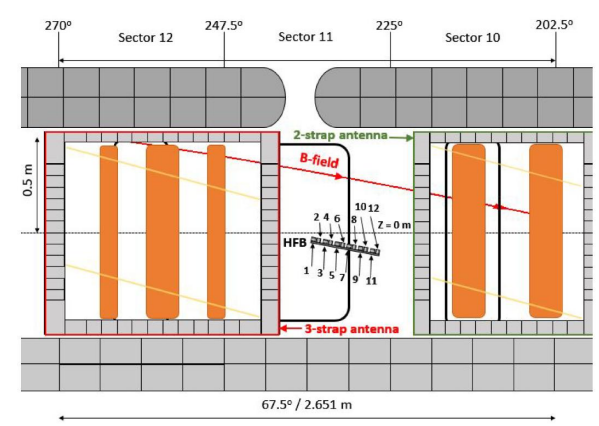

Fig.5: Placement of High frequency B-dot (HFB) probes

Like in the previous antenna, RF current shunts at selected limiter tiles [5], as well as temperature sensors were specified for the new antenna.

\section{Infrastructure}

Due to new concept of antennas, which offers the (needed) flexibility in feeding center strap vs. outer straps, installation of additional transmission lines and tuning elements were required, as shown in Fig.6. In addition ICRF Generators and their control and diagnostics system required complex enhancements to support the new functionality. These tasks were in the responsibility of IPP.

Trial installations in test environments as well as final installation and commissioning were joint activities supported by specialists from the project partners.
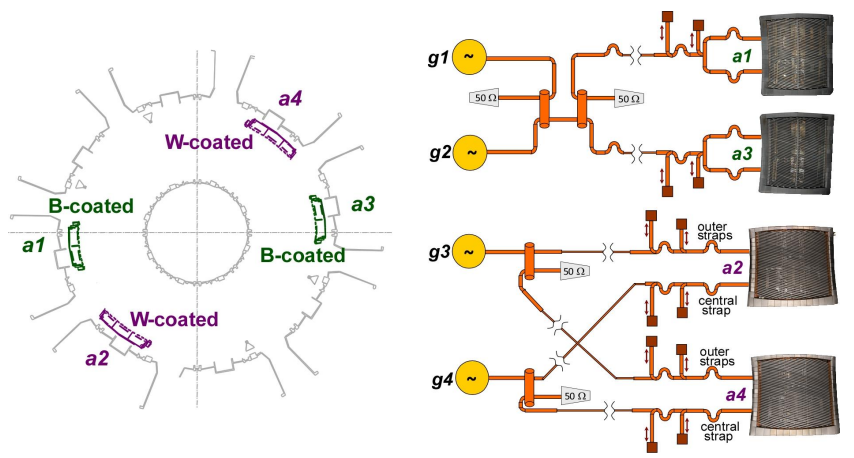

Fig. 6: Modified antenna arrangement and infrastructure, which now allows the feeding of the central and outer straps of antenna 2 and 4 independently in amplitude and phase (through generator 3 and 4).

\section{Material selection and process qualification}


Stainless steel with low permeability and Co content was specified for the main structures. $316 \mathrm{LN}$ (equivalent 1.4429) in excellent quality was sourced by ASIPP on the local market. A controlled batch of material, with verified chemical composition, having passed mechanical stress test was used for electron-beam welding qualification and later manufacturing.

For the cooling frame, where good thermal conductivity, adequate mechanical strength, vacuum compatibility are mandatory, $\mathrm{CuCrZr}$ was the material of choice. As a first batch failed the EB-welding tests, forged material was used for process validation samples. After having passed hot leak test, samples went into cycled pressure jump test (0-22 bar). No leakage was found in hot leak tests after 4500 cycles and excessive 300 bar pressure test resulted in deformation, but no leaks. To be prepared, repair methods were defined and validated upfront. This paid off during a later stage of project.

For the Faraday screen, Titanium carbide coated TZM (Titan-Zirconium-Molybdenum) rods were the material of choice. Besides qualification of the machining and bending processes, Ti-C coating was qualified by irradiating samples in GLADIS (Garching Large Divertor Sample Test Facility), followed by a bend and chip-off test.

For the limiters, Graphite coated with Tungsten, from a supplier previously qualified for AUG, was chosen.

\section{Validation}

After completion of the manufacturing drawings and the qualification of the associated manufacturing processes, the teams met for a final design review and the manufacturing release. The component, when they were reported to be ready, had to undergo a stringent factory acceptance test at either the manufacturer or the project partner supplying the component. The manufacturing and QA documentation were reviewed and tests of essential parameters like dimensions, surface treatments or vacuum tightness were witnessed.

The cooling frame, separating vessel vacuum from cooling water, required special attention. Following EBwelding of $\mathrm{CuCrZr}$ made cooling frame segments, leaks were found during Helium cold leak test performed by IPP team at manufacturers premises. Thanks to a staggered repair strategy, qualified upfront, all cooling frame segments could be repaired on a relatively short notice. After having passed cold leak test, each segment was high pressure He hot leak tested at IPP. Following orbital welding of the cooling water interconnections, the completely assembled cooling frames were again high pressure He hot leak tested.

\subsection{HV-RF test in Vacuum}

To test RF the high voltage handling capability, the first antenna was mounted in IPP's ICARoS test stand (a large vacuum vessel), matched with the help of stub tuners and loaded with RF power. Without plasma, the pass/fail criteria " $40 \mathrm{kV}$ in vacuum without arcing" was met at $300 \mathrm{~kW}$ input $\mathrm{RF}$ power, for more than 500 pulses.

\subsection{Trial installation}

Before final installation of the 3-strap antennas in the ASDEX Upgrade torus, one of the antennas was mounted in a full scale 1/8-segment (Octant) of AUG as shown in Fig. 7 to test the mechanical compatibility with AUG in-vessel components as well as to exercise assembling the antenna in a congested environment. This helped to iron out some mechanical conflicts that would have jeopardized the installation schedule, if only noticed during final installation in AUG vessel.

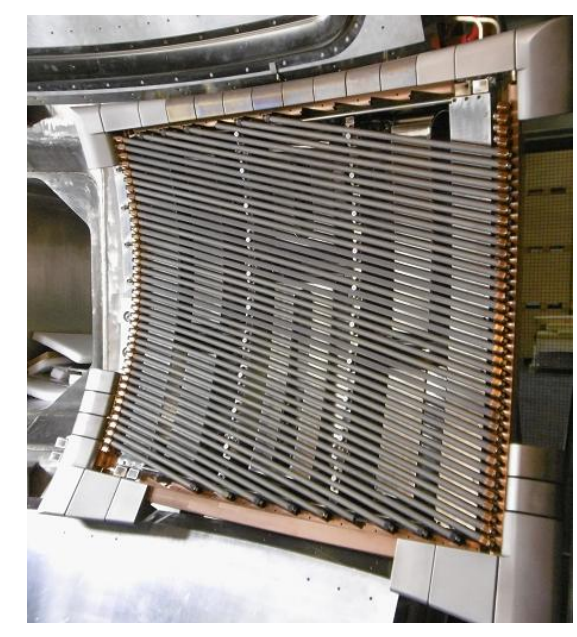

Fig. 7: First antenna during trial installation

\subsection{Near field measurement}

The octant installation, mentioned above, also presented an opportunity to perform low power $(\sim 10 \mathrm{~W}, 30 \mathrm{MHz})$ near-field RF tests measured directly in front of the 3strap antenna Faraday screen. Two series of tests were conducted. First, the strength of the excited RF magnetic fields (in the toroidal direction) was measured as a function of the RF current phasing and amplitude ratio between the central and the side straps, with the help of a B-dot probe [6]. The power and phasing ratio setting for a minimum at antenna left bottom corner then was used to scan the entire density distribution with the help of a $\mathrm{RF}$ field radiation meter.

\subsection{Reflectometry trial installation and test}

All the components of the reflectometer's transmission system (DC breaks, low pass filters, WR19 fundamental waveguides, WR42 truncated waveguides and WR19 rectangular horns) have been tested individually and then tested again during all the steps of their integration. The whole transmission lines have been integrated and installed in a mock up of an Octant of AUG, in order to guarantee their mechanical compatibility and to optimize 
the procedure for the final installation in AUG. Losses and reflections of all channels have been measured. An estimation of the level of coupling has been assessed in order to confirm that the amplitude of the signal reaching the mixer was adequate and to evaluate the impact of multiple reflections. This test was done by placing a stainless steel plate in front of the horns of different channels, with the metallic plate moved back from the horns in a controlled way. After the final installation in AUG, these tests were repeated.

\section{Installation in AUG}

After both antennas were completely trial assembled, one of them in the Octant and the other one in an equivalent mounting structure, including 3D measurements with the help of a FARO-Arm, both antennas were disassembled, the parts were carefully cleaned and bagged. The reason for disassembling is the very limited size of the access port, as shown in Fig.8.

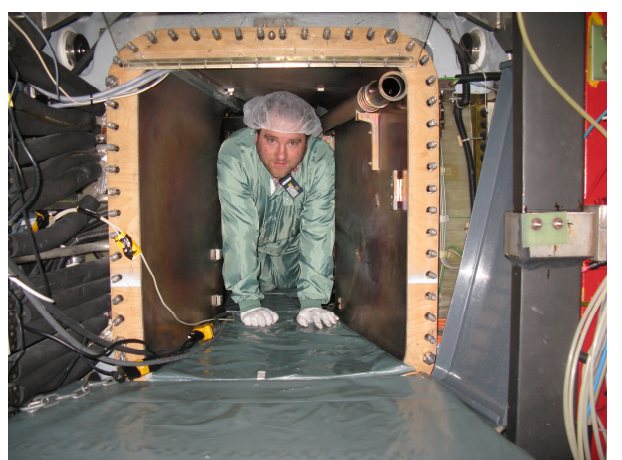

Fig. 8: The only access point for staff, materials and tools

Installation started with bringing in the segmented return conductor, followed by installation of the waveguides to the rear of it. To use the available invessel time to the best possible extent, a staff of two was allocated inside the vessel for each antenna, while on the outside a staff of two ensured flow of materials and tools. Due to the complexity of the work, partially in a very congested environment, installation of both antennas, one of them including reflectometry wave guide installation and test, this activity required about 8 weeks, almost two weeks less than allocated for. Fig.9 shows one of the installed new 3 strap antennas in the foreground, with one of the remaining 2 strap antennas in the background.

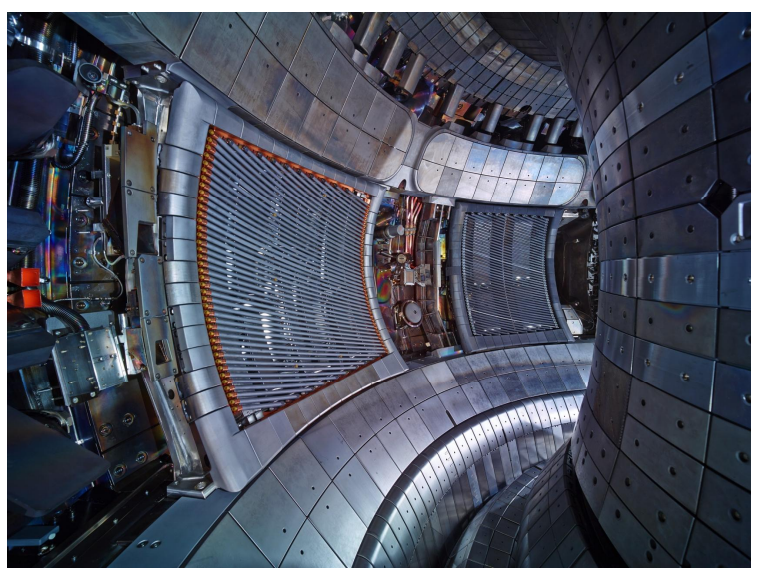

Fig. 9: Installed new 3 strap antenna in the foreground

\section{Test results of new antennas}

The new antennas show a significant reduction of $\mathrm{W}$ impurity production $[3,4]$ consistent with predictions [5].The reduction of the $\mathrm{W}$ content in the plasma is shown in Fig. 10.
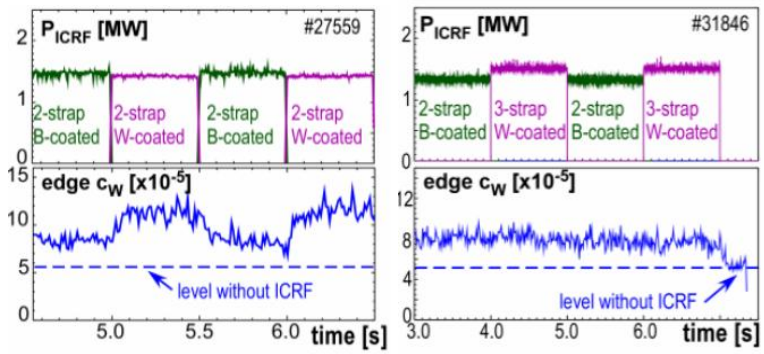

Fig. 10: W concentration: Left W-coated 2 strap vs. B-coated 2 strap, Right: W-coated 3 strap vs. B-coated 2 strap

\section{Reflectometry, installation and test results}

Before closing the AUG vessel in May for the 2015 campaign, distance calibration of the installed wave guide trains was done with the help of a reflective plate and lab type test equipment, Delays in the assembly of the electronic components of the multi channel reflectometry diagnostic only allowed the start of its use in November 2015, already during the operational campaign. Three of the ten available observation points, as shown in Fig. 11, were connected, allowing the measurement of edge plasma electron density profiles in front of the lower, middle and upper parts of the radiating surface of the ICRF antenna. The first plasma discharges after full installation were used to set the necessary gain for the reflection signal levels, the best configuration of probing sweep time and the required trigger delays. The density profile inversion codes from the raw data have been developed [7] and the new diagnostic fully commissioned. 


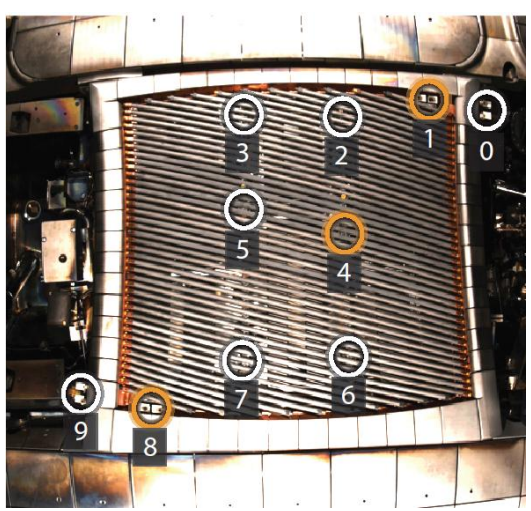

Fig. 11: Instrumented reflectometry locations 1,4 and 8

The new X-mode reflectometry diagnostic measures edge plasma electron densities from zero up to $2 \times 10^{19} \mathrm{~m}$ 3 , in magnetic fields between $1.5 \mathrm{~T}$ and $2.7 \mathrm{~T}$, using probing frequencies in the $40-68 \mathrm{GHz}$ band. The different probing locations enable the study of local ICRF power interactions with the plasma, such as convective transport during heating operation. Fig. 12 shows the local evolution of the constant-density layers during an ELM event, demonstrating the poloidal resolution provided by this unique installation of a multichannel reflectometry system in an ICRF antenna. Its capability will support the analysis of poloidal variations during ICRF operation, like RF induced convective cells.

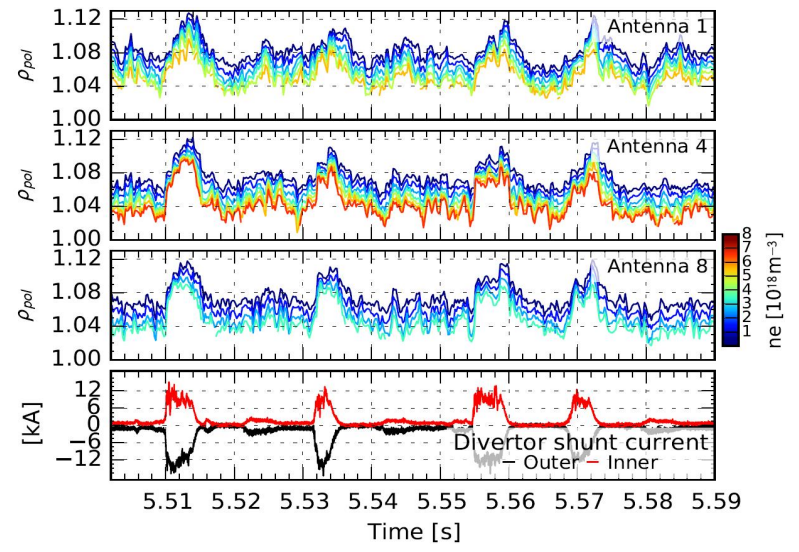

Fig. 12: Constant density layers during an ELM event

\section{Summary and lesson learned}

The first antenna (the stainless steel components) passed the on-site factory acceptance test (FAT) in June 2013, while $2^{\text {nd }}$ antenna arrived at IPP in April 2014. Following assembly with the special material parts, delivered by ENEA in summer 2014, the antennas were pre-installed and tested in a full scale AUG segment during the fall of 2014. Following the opening of AUG, two old antennas were de-installed in December 2014.

Both new antennas were installed in AUG in the reserved time slot from mid of February to end of April 2015. AUG experiment, permitting commissioning and evaluation of new antennas started again in June 2015. The overall project time line is shown in Fig. 13.

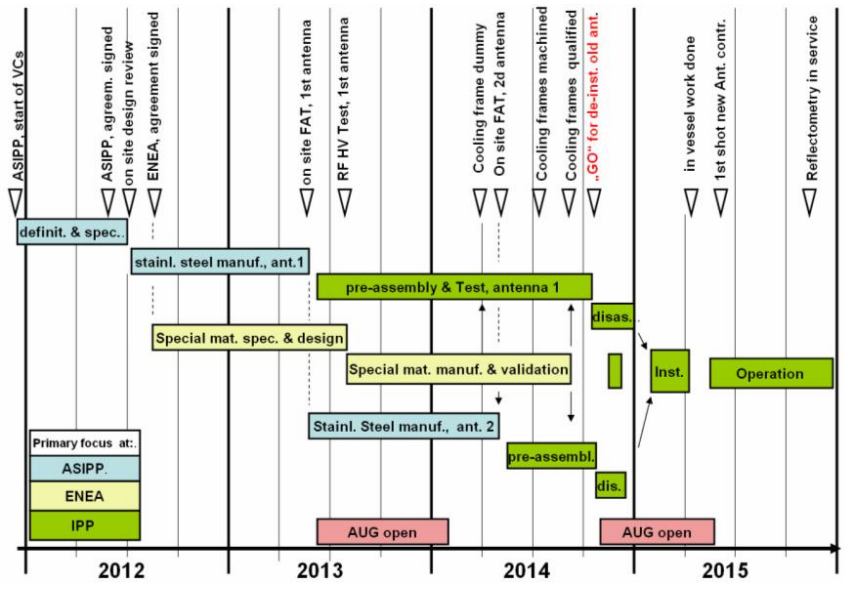

Fig. 13: Project timeline

A total of 166 video conferences were held and documented, with about 350 higher level action items dealt with. Application of milestone trend analysis proved to be a valuable tool to visualize arising problems in an early stage.

Qualification of material and manufacturing processes took longer than expected and some of the specified tolerances appeared to be very challenging, considering that there was neither funding nor the schedule reserved for a complete prototyping.

Thanks to the dedication and team spirit of all involved, this multinational project, involving four institutes in four countries on two continents, uncounted subcontractors and suppliers, despite all technological and technical challenges and programmatic boundaries, was successfully completed from start to operational service within three years and was a great experience for the core team, shown on Fig. 14.

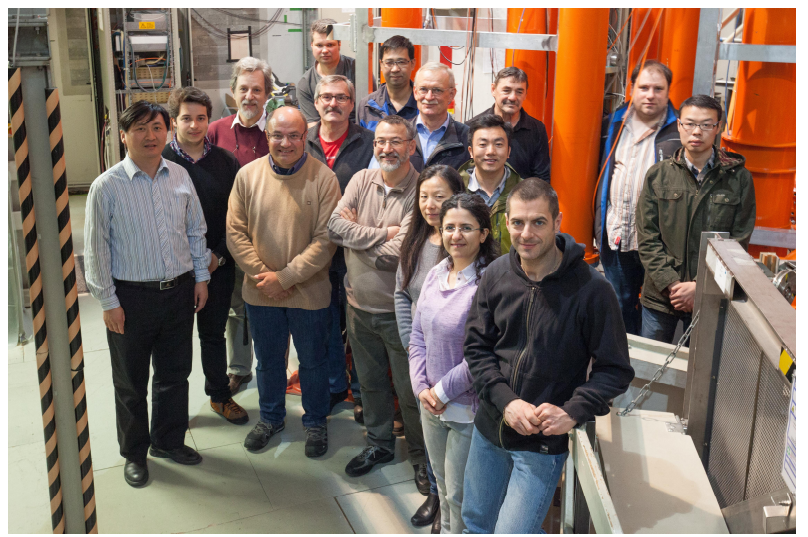

Fig. 14: The multinational core team

\section{Outlook}

As a consequence of required power ratio from central strap to outer strap, the RF Generator feeding the outer straps can not be used to its maximum power. To allow full, even increased power operation, an additional 2MW 
RF generator (in the past used by the ASDEX and Wendelstein 7-AS experiments and recently modified to use a modern tetrode) is in the process of being integrated into AUG's ICRF system, as shown in Fig. 15.

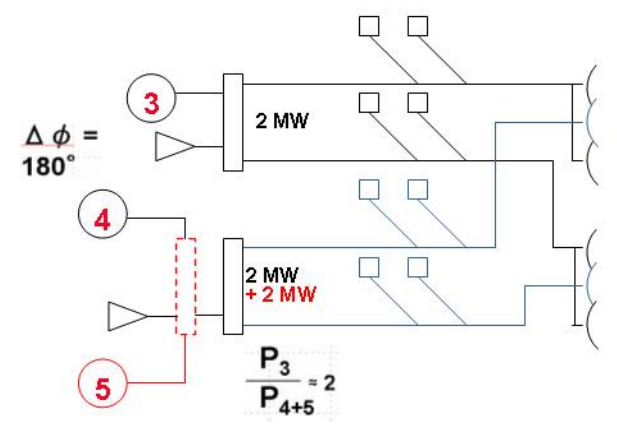

Fig. 15: Additional 2MW installed ICRF power

After above enhancement, bringing the installed ICRF power on AUG to a total of 10MW, follow on steps under consideration are either to replace the two remaining 2-strap antennas by 3-strap antennas, including adding a further $2 \mathrm{MW}$ generator, or the implementation of a distributed antenna with low mechanical depth.

\section{Acknowledgments}

This work has been carried out within the framework of the EUROfusion Consortium and has received funding from the Euratom research and training programme 2014-2018 under grant agreement No 633053. The views and opinions expressed herein do not necessarily reflect those of the European Commission.

\section{References:}

[1] V. Bobkov et al., Assessment of compatibility of ICRF antenna operation with full $\mathrm{W}$ wall in ASDEX Upgrade, 2010 Nuclear Fusion $\mathbf{5 0} 035004$

[2] V. Bobkov et al., ICRF operation with improved antennas in ASDEX Upgrade with W wall, 2013 Nuclear Fusion 53 09318

[3] V. Bobkov et al., First results with 3-strap ICRF antennas in ASDEX Upgrade, Nuclear Fusion 56084001

[4] J.-M. Noterdaeme et al., , Ion Cyclotron Range of Frequency power Challenges and solutions „26th IAEA Kyoto - 2016

[5] V. Bobkov et al., „Making ICRF Power compatible with a high-Z wall in ASDEX Upgrade“", EPS 2016, submitted to Plasma Physics and Controlled Fusion.

[6] R. Ochoukov et al., „, A new B-dot probe-based diagnostic for amplitude, polarization and wavenumber measurements on ion cyclotron range of frequency fields on ASDEX Upgrade, Review of Scientific Instruments 86, 115112 (2015)

[7] D. Aguiam et al., „Implementation of the new multichannel $\mathrm{X}$-mode edge density profile reflectometer for the ICRF antenna on ASDEx upgrade“, Review of Scientific Instruments 87, 11E722 (2016)

[8] D. Aguiam et al., „U-band X-mode raw data analysis for edge density profile measurements using the new ICRF antenna reflectometer on ASDEX upgrade“, SOFT 2016 